\title{
Intravitreal Injections of Melanocortin Receptor 1, 5 Agonists Prevents Neovascularization in a VEGF165 Mouse Model of Retinopathy
}

\author{
Rosa Maisto ${ }^{1}$, Maria Consiglia Trotta ${ }^{1}$, Settimio Rossi ${ }^{2}$, Carlo Gesua \\ Francesca Simonelli ${ }^{2}$, Clara Di Filippo ${ }^{1}$, and Michele D' Amico, ${ }^{1, *}$ \\ ${ }^{1}$ Department of Experimental Medicine, University of Campania “Luigi Vanvitelli”, Italy \\ ${ }^{2}$ Multisciplinary Department of Medical-Surgical and Dental Specialities, University of Campania “Luigi Vanvitelli”, Italy \\ ${ }^{3}$ Department of Clinical, Public and Preventive Medicine, University of Campania "Luigi Vanvitelli", Italy \\ ${ }^{4}$ School of Medicine, Catholic University of Valencia, Spain
}

${ }^{*}$ Correspondence to: Michele D’Amico, Department of Experimental Medicine, University of Campania “Luigi Vanvitelli", Italy, Tel: +39-081-566-5920;

Email: michele.damico@unicampania.it

Received: April 05, 2018; Accepted: April 13, 2018; Published: April 20, 2018;

\begin{abstract}
Background: The present study focuses on the evaluation of the protective effect on retinal neovascularization exerted by melanocortin receptor $1-5$ (MCR 1-5) agonists in a mouse model of proliferative retinopathy induced by intravitreal injection of vascular endothelial growth factor 165 (VEGF165).

Methods: Intravitreal injections of the MCR1 agonist BMS-470539 and of the MCR5 agonist PG-901 were performed 7 days before the VEGF165 injection and 1 day after VEGF165 injection. Fluorescein angiography (FAG), immunohistochemical and biochemical analysis were performed at different time points during the follow up.

Results: FAG showed a marked vascular tortuosity accompanied by retinal neovascularization in mice receiving VEGF165 intravitreal injection and the absence of neovascularization in mice treated with MCR 1-5 agonists, confirmed by CD34 antigen immunostaining. The biochemical analysis of retinal VEGFR2 gene and protein expression showed that MCR 1-5 agonists significantly reduced VEGFR2 levels, increasing in parallel retinal mir-150 expression.
\end{abstract}

Conclusions: According to these evidences, we propose that intravitreal injections of melanocortin receptor agonists up-regulate mir-150 expression, and this consequently reduces VEGFR2 expression levels with the endpoint being the blockade of VEGF165 induced retinopathy.

Keywords: intravitreal injection; neovascularization; melanocortin receptors; retina; VEGF;

\section{Introduction}

Some of the major eye diseases that cause blindness worldwide are related to intraocular neovascularization. Among these conditions we must consider age-related macular degeneration (AMD), retinopathy of prematurity (ROP), diabetic retinopathy (DR) and retinal vascular occlusions (RVO), which are all linked to ocular ischemia that causes progressively elevated intraocular levels of vascular endothelial growth factor (VEGF) with secondary retinal, subretinal or intravitreal neovascularization leading to progressive visual impairment [1-4].

VEGF is the main player in neovascularization during proliferative retinopathy, it plays a key role as a potent inducer and modulator of proliferation, permeability and survival of endothelial cells. Four VEGF proteins are known VEGFA, -B, -C, -D that act through interaction with the VEGFR1, -2 , and -3 receptor subtypes $[5,6]$. Among these, VEGFR2 is the main receptor that modulates endothelial cell proliferation, migration and in vivo angiogenesis $[5,6]$. Instead, VEGF-C and -D are involved in the modulation of cell adhesion and cell migration and in extracellular matrix degradation; VEGF-B and PIGF are involved in lymphangiogenesis [7]; VEGF-A promotes the process of neovascularization and increased vascular permeability. In particular, VEGF164(165) isoform is primarily implicated in the pathogenesis of major eye diseases that recognize ocular ischemia as a prime mover [8-11].

The introduction in clinical practice of various anti-VEGF drugs injected intravitreally (bevacizumab, ranibizumab, aflibercept) [12] for the management of the main eye diseases has positively changed the evolution and prognosis of diseases such as AMD,ROP, DR and RVO. In fact, an open research of new strategies and tools has been undertaken nowadays.

Previous studies by Rossi et al.,(2016) indicated that one of these strategies could be that of the modulation of the activity of the retinal endogenous melanocortin pathway and receptors. Indeed, he showed that an agonism at melanocortin receptor 1,5 (MCR1-5) attenuates retinal damage in a mouse model of streptozotocin (STZ) induced 
diabetic retinopathy $(\mathrm{DR})$ by modulating the pattern of cytockine and chemochine expression [13]. Subsequently, Maisto el al. evidenced that this MCR1-5 agonism restores antioxidant enzyme levels in high glucose primary retinal cells and protects the photoreceptors from the glucose induced damage [14]. These receptors, therefore, appeared to be good candidates for targeted therapy of diabetic retinopathy and possibly its proliferative form as well. Noteworthy, these melanocortin receptors have already been accredited to control a wide range of biological activities including inflammation, oxidative stress, energy homeostasis, sexual function, pain and immune response [13].

Based on this experience, the present study aimed to evaluate the protective effect exerted by MCR 1-5 agonists on retinal vascularization in a mouse model of retinopathy induced by intravitreal injection of VEGF165.

\section{Materials and Methods}

BMS-470539 and PG-901 were used as MCR1 and MCR5 agonist respectively [15] and were supplied by Professor Grieco (Pharmacy Department, University of Naples Federico II).

\section{Animals}

All the experimental procedures were performed according to Animal Care Ethical Committee of the University of Campania, in accordance with ARVO Statement for the use of Animals in Ophthalmic and Vision Research.

C57BL/6 mice (Harlan, Italy) aged 7 to 10 weeks were housed in standard cages with 24-hours light-dark cycle, humidity and temperature automatically controlled. C57BL/6 mice were divided into 2 settings: the first setting was composed of 2 groups ( $\mathrm{n}=12$ animals per group), treated respectively with sham intravitreous injections of $5 \mu \mathrm{l}$ balanced saline $(0.1 \mathrm{ml})$ and $5 \mu \mathrm{l}$ intravitreal injection

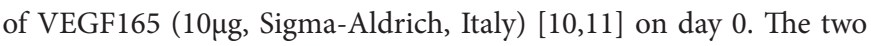
groups were monitored by FAG in order to ascertain the time course of the vascular modifications. The second setting was composed of the following 2 groups ( $n=8$ animals per group): (1) mice treated with $5 \mu \mathrm{l}$ intravitreal injection of the MC1 receptor agonist BMS-470539 (33 $\mu \mathrm{mol}$ ) [16] 7 days before the injection of VEGF165 and 1 day after VEGF165 injection; (2) mice treated with $5 \mu \mathrm{l}$ intravitreal injection of the MC5 receptor agonist PG-901 (7.32 nM) [17] 7 days before the VEGF165 injection and 1 day after VEGF165 injection, following pioneering in house experience.

\section{Intravitreal injections}

Mice were anesthetized by pentobarbital ( $45 \mathrm{mg} / \mathrm{kg}$ in saline i.p.). Tropicamide (5\%) was instilled into the right eye of each animal, in order to induce papillary dilation, and tetracaine (1\%) was topically applied for local anaesthesia. Physiological saline, VEGF165 or MC receptor agonists (5 $\mu \mathrm{L}$ volume) were injected intravitreally into the right eye using sterile syringes fitted with a 30-gauge needle (Microfine; Becton Dickinson AG, Meylan, France), as previously described $[10,11,18]$. Briefly, a transconjunctival 30-gauge, 1.5-mm needle was introduced behind the limbus in the superior temporal quadrant. When the needle was visualized at the vitreous, the various substances were injected in the various experimental groups. After the needle was withdrawn, the scleral entrance site was compressed with a cotton tip for $1 \mathrm{~min}$ and a topical antibiotic ointment was applied.

The following MC receptor ligands were used at the indicated doses as selected from the reported publications: BMS-470539, 33 $\mu$ mol; PG-901, $7.32 \mathrm{nM}[16,17]$.

\section{Fluorescein Angiography (FAG)}

For FAG assessment the animals were restrained without anesthesia by grasping firmly at the base of the tail and grasping the scruff of the neck. FAG was performed by using a Topcon TRC-50DX apparatus (Topcon, Japan) following intraperitoneal injection of $10 \%$ fluorescein sterile solution $(1 \mathrm{~mL} / \mathrm{kg}$ body weight, AK-Fluor; Akorn Inc., USA). Fundus photographs were captured in order to display the retinal vasculature and to evaluate the early typical alterations of microangiopathy.

\section{Follow-up}

In the first setting, FAG was performed after papillary dilatation on all the animals receiving the intravitreal injection of balanced saline or VEGF165 in order to detect the appearance of early vascular disorders. At 7 the day time point, when FAG evidenced neovascularization in VEGF165 mice, 4 animals out of the 12 for each group were randomly chosen, sacrificed and assayed for VEGFR2 and other biochemical parameters. The remaining 8 animals were monitored with FAG at the 14 day time point, when neovascularization was still evident. Again, 4 mice out of the 8 were randomly chosen, sacrificed and assayed for same biochemical parameters as at 7 day time point. The remaining 4 animals were monitored by FAG at 28 days, when no signs of neovascularization were evidenced.

In the second setting, mice receiving intravitreal injection of BMS470539 and PG-901 seven days prior and one day after VEGF165 were monitored by FAG at 7 and 14 day time points. At 7 days, 4 animals out of the 8 for each group were randomly chosen, sacrificed and assayed for VEGFR2 and other biochemical parameters. The remaining 4 animals were sacrificed and assayed for same biochemical parameters at 14 day time points.

For the biochemical analysis, the eyes were displaced forward by placing curved forceps around the posterior part and cut in two halves. On one half of each eye the cornea was cut using a sharp blade or scalpel, and the retina was squeezed through the cut together with residual pigment epithelium and lens by applying gentle pressure with the forceps. Dissected retina was placed in cooled PBS, freed from non retinal tissue using the forceps, and immediately frozen in liquid nitrogen and stored at $-80^{\circ} \mathrm{C}$ for subsequent biochemical analysis. The other half of each eye was fixed by immersion in $10 \%$ neutral buffered formalin and paraffin-embedded for immunohistochemistry.

\section{Immunohistochemistry}

CD34 antibody was used as marker of neo-angiogenesis (sc-74499 Santa Cruz Biotec, USA). The ocular tissues were incubated with the primary antibody, washed in PBS, and incubated with secondary antibody. The ocular samples were analyzed by an expert pathologist (variability 6\%); every sample was visualized at 200x magnification. 
The number of CD34 positive particles per area was analyzed in 20 microscopic fields and expressed as percentage of positive stained area/total area.

\section{Total RNA isolation; mir-150 and VEGFR2 mRNA expression levels}

The extraction of total RNA, including small RNAs, was performed according the MiRNeasy Minikit protocol (Qiagen, Italy). Syn-celmiR-39 miScript miRNA Mimic $5 \mathrm{nM}$ (Qiagen, Italy) was added to each sample before the extraction as internal control. The total RNA concentration and integrity were determined by Nanodrop ND1000 UV spectrophotometer (Nano-Drop ${ }^{\circledR}$ Technologies, Thermo Scientific, USA).

The qRT-PCR analysis for mir-150 was performed on Mastercycler personal (eppendorf, Germany) using miScript II Reverse Transcription Kit (Qiagen, Italy) and on CFX96 Real Time

System cycler (BioRad, Italy) using miScript SYBR Green Kit (Qiagen, Italy) and miScript Primer Assays specific for Syn-celmiR-39 and mir-150 (Qiagen, Italy).

VEGFR2 gene expression was measured by RT-PCR amplification on Mastercycler personal (eppendorf, Germany). cDNA synthesis was obtained using SuperScript III Reverse Transcriptase Kit (Invitrogen, USA) starting from $200 \mathrm{ng}$ of total RNA. Aliquots of $2 \mu \mathrm{lDNA}$ were transferred into a $25 \mu \mathrm{l}$ PCR reaction mixture containing dNTPs, $\mathrm{MgCl} 2$, reaction buffer, specific primers and GoTaq Flexi DNA polymerase (Promega, USA). Sequences for the mouse VEGFR2 mRNA from GeneBank (DNASTAR INC., USA) were used to design specific primer pairs for RT-PCR (Microtech, Italy). The housekeeping gene chosen was hypoxanthine-guanine phosphoribosyl transferase (HPRT). PCR products were resolved into $2.0 \%$ agarose gel. A semiquantitative analysis of VEGFR2 mRNA levels was carried out by the Gel Doc EZ UV System (Bio-Rad, USA).

Relative quantification of gene expression was normalized to Syncel-miR-39 for mir-150 and to HPRT for VEGFR2, using the $2^{\wedge}-$ $\Delta \Delta \mathrm{Ct}$ method.

\section{Enzyme-Linked Immunosorbent Assay (ELISA)}

Mouse VEGFR2/Flk-1 Quantikine ELISA Kit (R\&D System, UK) was used in the homogenate of dissected retina in order to assess the ocular protein VEGFR2 expression levels, according to the manufacturer's protocol.

\section{Statistical Analysis}

All values are expressed as mean \pm standard error of the mean (SEM) of $=4$ mice. Statistical analyses were assessed either by Student's -test (when only two groups were compared) or one-way analyses of variance (ANOVA), followed by Dunnett's post hoc test (more than two experimental groups). $<0.05$ was considered statistically significant.

\section{Results}

Melanocortin Receptor1-5 activation prevents the development of retinal neovascularization

No significant vascular alterations were observed in the control group at any of the various time points (Figure 1a and Figure 2a). All the mice treated only with intravitreal injection of VEGF165 developed vascular tortuosity accompanied by retinal neovascularization at 7 days that persisted up to 14 days post-treatment, while it disappeared at the day 28 time point (Figure $1 \mathrm{~b}$ and Figure $2 \mathrm{~b}$ ).

a
7 days

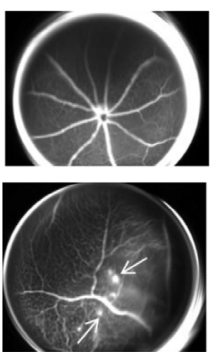

14 days

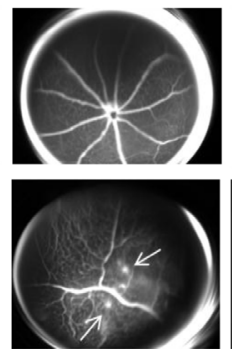

28 days

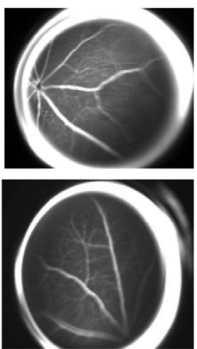

Control

VEGF165

Figure 1. (a) Representative FAG images of a control eye. There are no vascular alterations at the various time points. (b) Representative FAG images of mice treated with intravitreal injection of VEGF165: a marked vascular tortuosity accompanied by retinal neovascularization is present at 7 days and persists until 14 days, while it disappears at the 28 day time point. Arrows indicate the formation of neovessels.

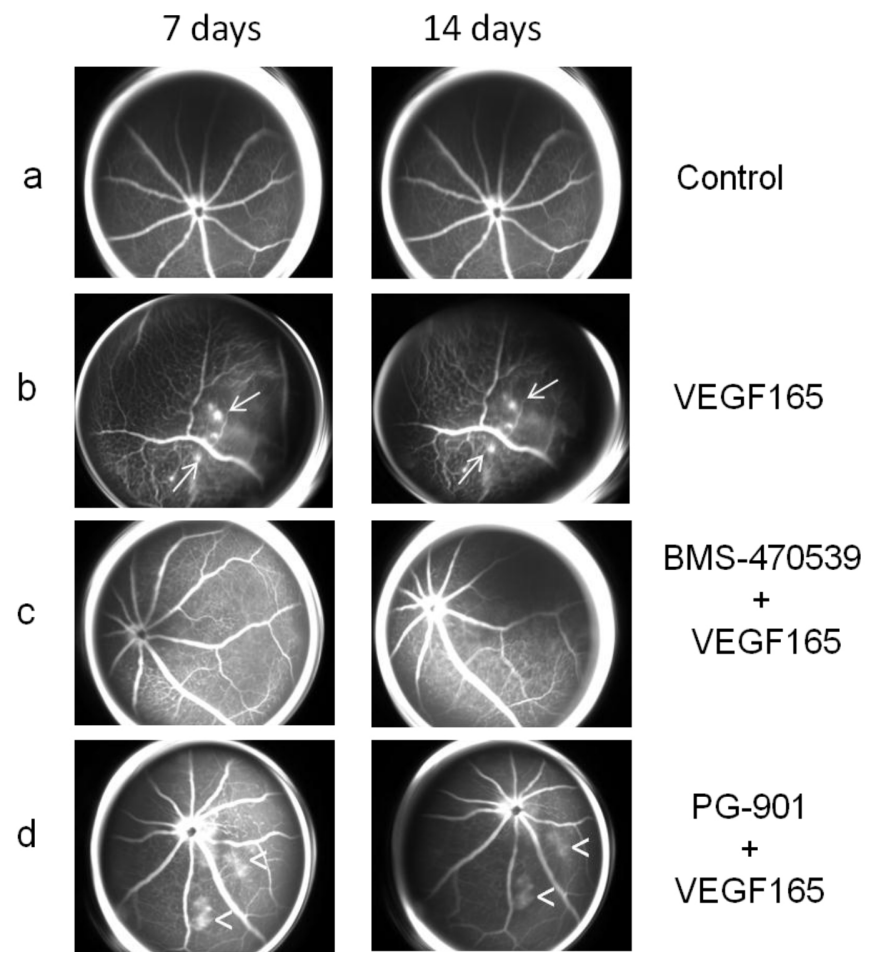

Figure 2. (a) Representative FAG images of a control eye (as Figure 1a). (b) Representative FAG images of mice treated with intravitreal injection of VEGF165 (as Figure 1b). (c) Representative FAG images of mice treated with intravitreal injection of BMS-470539 $(33 \mu \mathrm{mol}) 7$ days before and 1 day after the injection of VEGF165: the mice did not develop retinal neovascularization during the follow-up but only an irregular vessel caliber appeared at 7 days and persisted until the end of the follow-up at 14 days. (d) Representative FAG images of mice treated with intravitreal injection of PG-901 (7.32 nM) 7 days before and 1 day after the injection of VEGF165: the mice did not develop retinal neovascularization but only an irregular vascular course and caliber was observed, accompanied by an increase in vascular permeability, resulting in dye leakage evident at 7 days and persisting until the end of follow-up at 14 days. Arrows indicate the formation of neovessels; arrowheads indicate vascular leakage.

Intravitreal injections of MC1 receptor agonist BMS-470539 (33 $\mu \mathrm{mol})$ or MC5 receptor agonist PG-901 $(7.32 \mathrm{nM})$ protect retinal vasculature, as demonstrated by FAG. In particular, mice treated with 
BMS-470539 $(33 \mu \mathrm{mol}) 7$ days before and 1 day after the injection of VEGF165 did not develop retinal neovascularization during the follow-up, only an irregular vessel caliber appeared at 7 days and persisted until the end of the follow-up at 14 days (Figure 2c). The mice treated with PG-901 (7.32 nM) 7 days before and 1 day after VEGF165 injection did not develop retinal neovascularization during the follow-up, only an irregularity of the vascular course and caliber was observed. However, this was accompanied by an increase in the vascular permeability resulting in dye leakage which was evident at the 7 day time point and persisted until the end of follow-up at 14 days (Figure 2d).

\section{Melanocortin MC1,5 receptor agonists reduce the retinal expression of} the hematopoietic endothelial progenitor cell antigen CD34

In order to confirm the development of retinal neovascularization at 7 and 14 days after VEGF165 intravitreal injection, immunohistochemistry for CD34 was performed in 4 mice per group randomly chosen. At 7 and 14 days, the analysis showed a significant $(\mathrm{P}<0.01)$ increase of $\mathrm{CD} 34$ immunostaining in mice receiving intravitreal VEGF165 compared to the mice receiving saline solution (Figure 3-4). In contrast, a marked decrease in the percentages of CD34 positive stained area/total stained area was observed at both time points in retinal tissue of mice receiving intravitreal injections of the MC1 agonist BMS-470539 (40.0 $\pm 5.15 \%$ at 7 days; $31.8 \pm 4.3 \%$ at 14 days) or the MC5 agonist PG-901 (46.7 $\pm 4.7 \%$ at 7 days; $28.9 \pm$ $4.7 \%)$ as calculated against the values quantified in VEGF165 mice (Figure 3 and Figure 4).

\section{Melanocortin MC1,5 receptor activation decreases retinal VEGFR2 gene expression}

qRT-PCR analysis of VEGFR2 gene expression in the retina showed that in mice receiving a single intravitreal injection of BMS470539 and PG-901 7 days prior and first day after VEGF165, BMS470539 and PG-901 significantly down-regulate VEGFR2 gene expression levels both at 7 and 14 day $(\mathrm{P}<0.01)$ time points compared with the group receiving saline only (Figure $5 \mathrm{a}$ ).

\section{Melanocortin MC1,5 receptor activation increases mir-150 ex- pression levels}

Intravitreal injection of the MC1 agonist BMS-470539 and the MC5 agonist PG-901 7 days prior and first day after VEGF165, significantly up-regulate retinal mir-150 expression levels at 7 and 14 days $(\mathrm{P}<0.01)$ time points compared with the group receiving saline only (Figure 5b). The single injection of VEGF165 into the vitreous does not affect qRT-PCR analysis of the retina for mir-150 expression levels (Figure 5b).

\section{Intravitreal injection of melanocortin $\mathrm{MC1}, 5$ receptor agonists re- duces retinal VEGFR2 protein levels}

To confirm gene expression data, retinal VEGFR2 protein levels were measured by ELISA assay, and fitting with RT-PCR results, VEGRF2 protein levels significantly decreased after BMS-470539 and PG-901 ( $<<0.01$, Figure 6) at 7 and 14 time points.

\section{a}

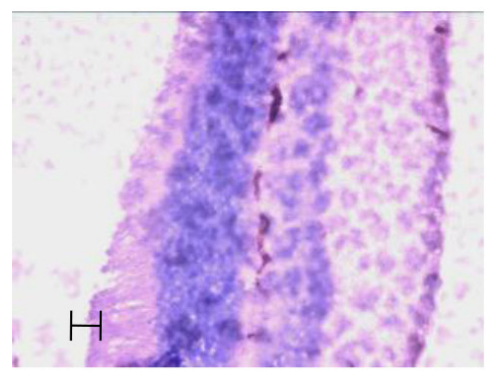

CTR

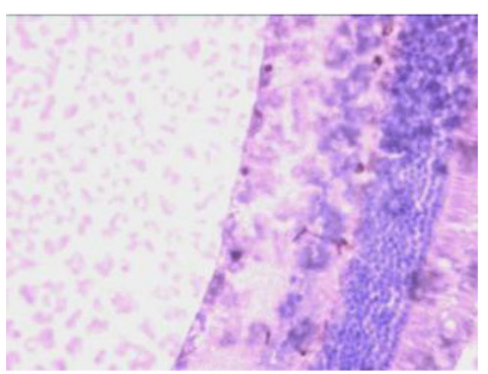

BMS-470539+ VEGF165

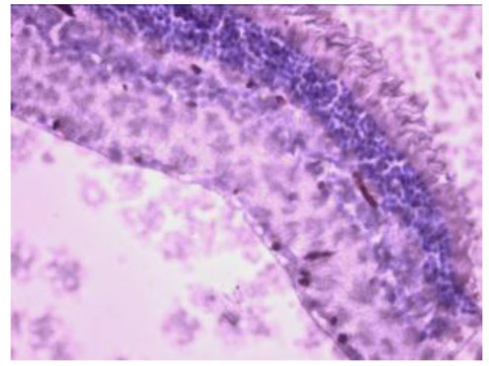

VEGF165

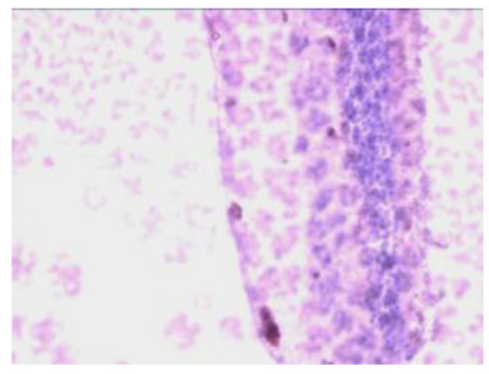

PG-901+ VEGF165 b

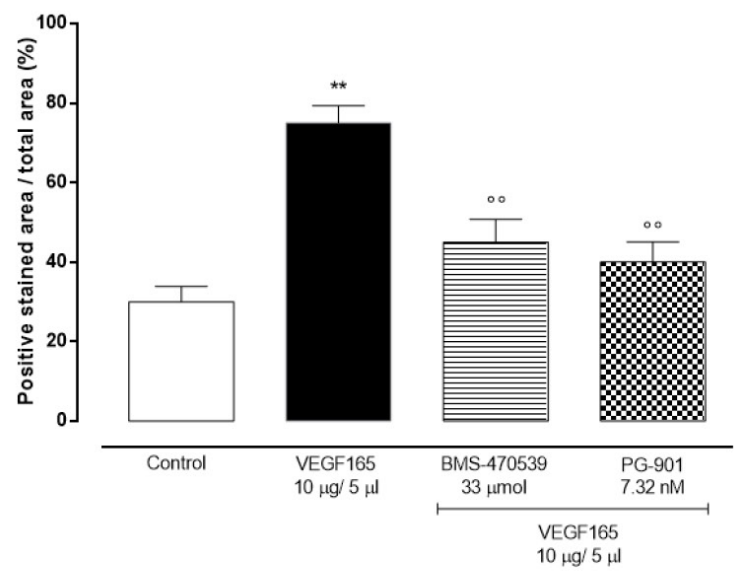

Figure 3. (a) Representative immunohistochemistry for CD34 in the retina of mice treated with vehicle, VEGF165(10 $\mu \mathrm{g} / 5 \mu \mathrm{l})$, BMS-470539 $(33 \mu \mathrm{mol})+\mathrm{VEGF} 165$ and PG-901 $(7.32$ $\mathrm{nM})+$ VEGF165, 7 days after VEGF165 intravitreal injection. BMS-470539 and PG-901 reduce CD34 immunostaining compared to rats receiving only VEGF165. Scale bar, 50 m. 200x magnification. (b) Graph showing the percentage of the total positive stained area for CD34 per total area analyzed at 200x magnification. Values are mean \pm SEM of $=4$ observations for each group. ** $\mathrm{P}<0.01$ vs Control; ${ }^{\circ} \mathrm{P}<0.01$ vs VEGF165. 
a

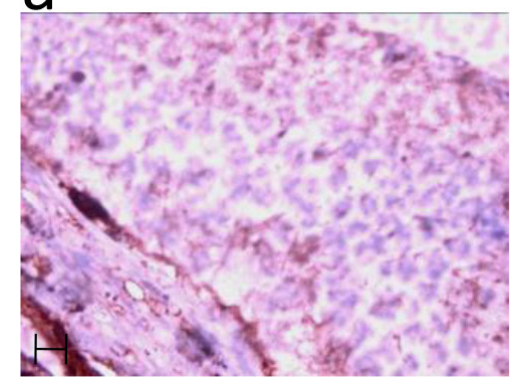

CTR

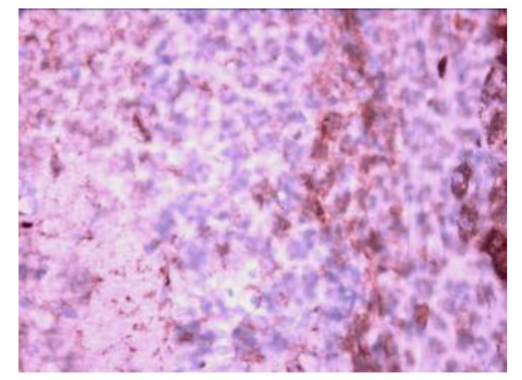

BMS-470539 + VEGF165

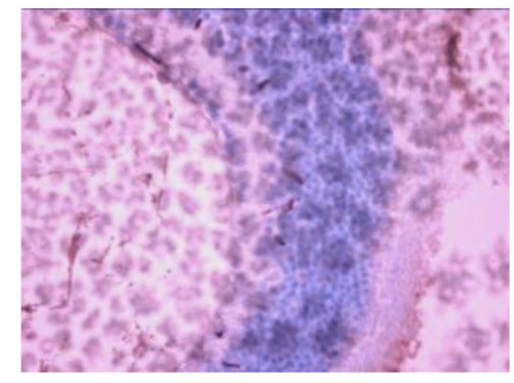

VEGF165

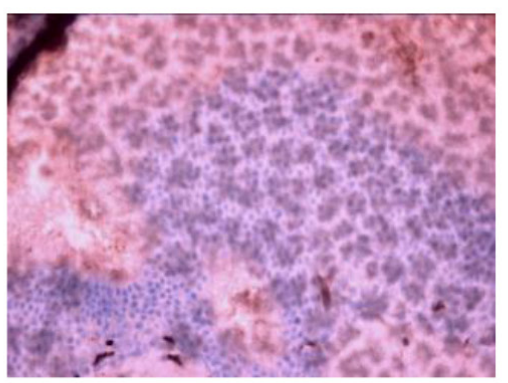

PG-901 + b

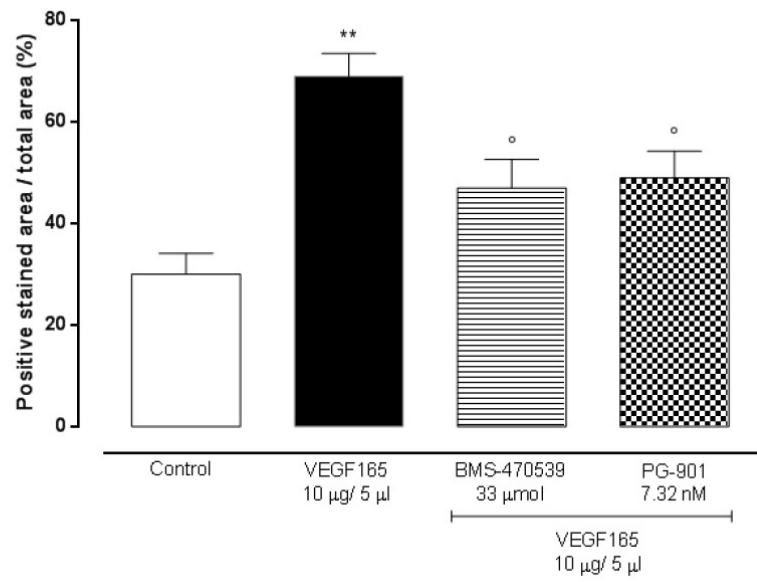

Figure 4. (a) Representative immunohistochemistry for CD34 in the retina of mice treated with vehicle, VEGF165(10 $\mu \mathrm{g} / 5 \mu \mathrm{l}), \mathrm{BMS}-470539(33 \mu \mathrm{mol})+\mathrm{VEGF} 165$ and PG-901 (7.32 nM) + VEGF165, 14 days after VEGF165 intravitreal injection. BMS-470539 and PG-901 reduce CD34 immunostaining compared to mice receiving only VEGF165. Scale bar, 50 m. 200x magnification. (b) Graph showing the percentage of the total positive stained area for CD34 per total area analyzed at 200x magnification. Values are mean \pm SEM of $=4$ observations for each group. ** $\mathrm{P}<0.01$ vs Control; ${ }^{\circ} \mathrm{P}<0.05$ vs VEGF165.

a
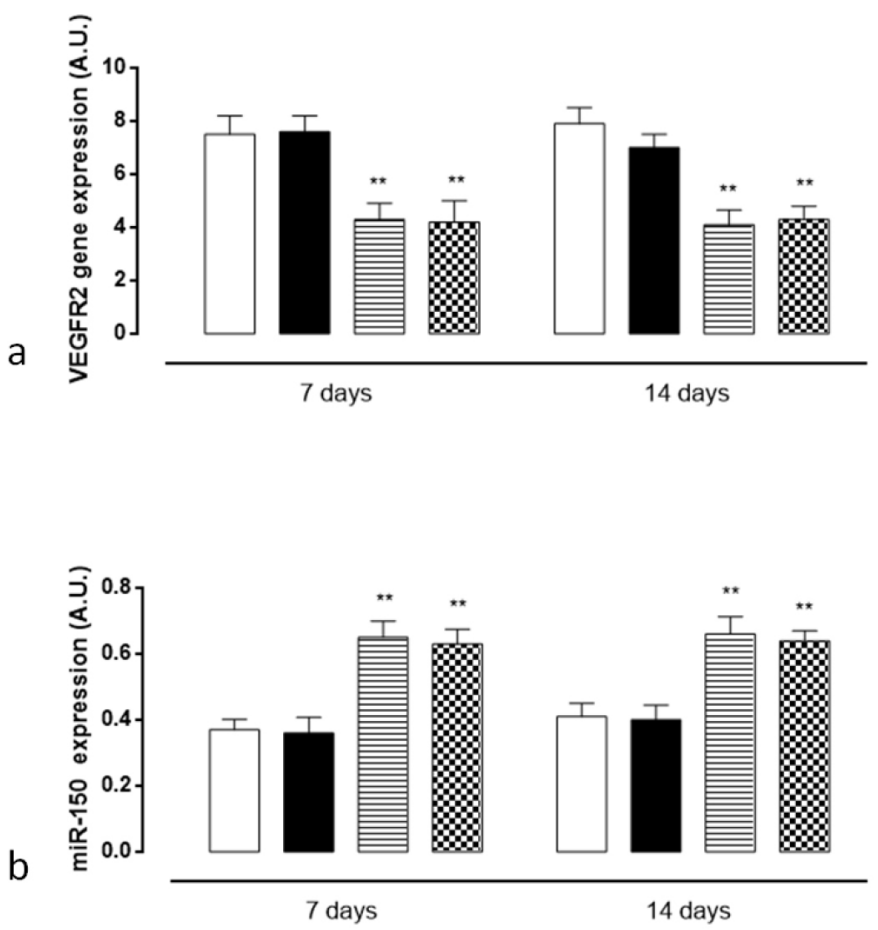

Figure 5. (a) RT-PCR analysis for VEGFR2 gene expression in retinal tissue 7 and 14 days after VEGF165 intravitreal injection. Treatment with BMS$470539(33 \mu \mathrm{mol})$ and PG-901 (7.32 nM) significantly decreases VEGFR2 mRNA expression levels at 7 and 14 days, compared to mice receiving saline only. Results are expressed as mean \pm SEM of $=4$ observations for each group. $* * \mathrm{P}<0.01$ vs Control. A.U. $=$ arbitrary units. (b) $\mathrm{qRT}$-PCR analyzing mir-150 expression in retinal tissue 7 and 14 days after VEGF165 intravitreal injection. At 7 and 14 days, BMS-470539 (33 $\mu$ mol) and PG-901 (7.32 nM) significantly increase mir-150 expression levels compared to mice receiving saline only. Values are mean \pm SEM of $=4$ observations for each group. ${ }^{* *} \mathrm{P}$ $<0.01$ vs Control. A.U. $=$ arbitrary units. 


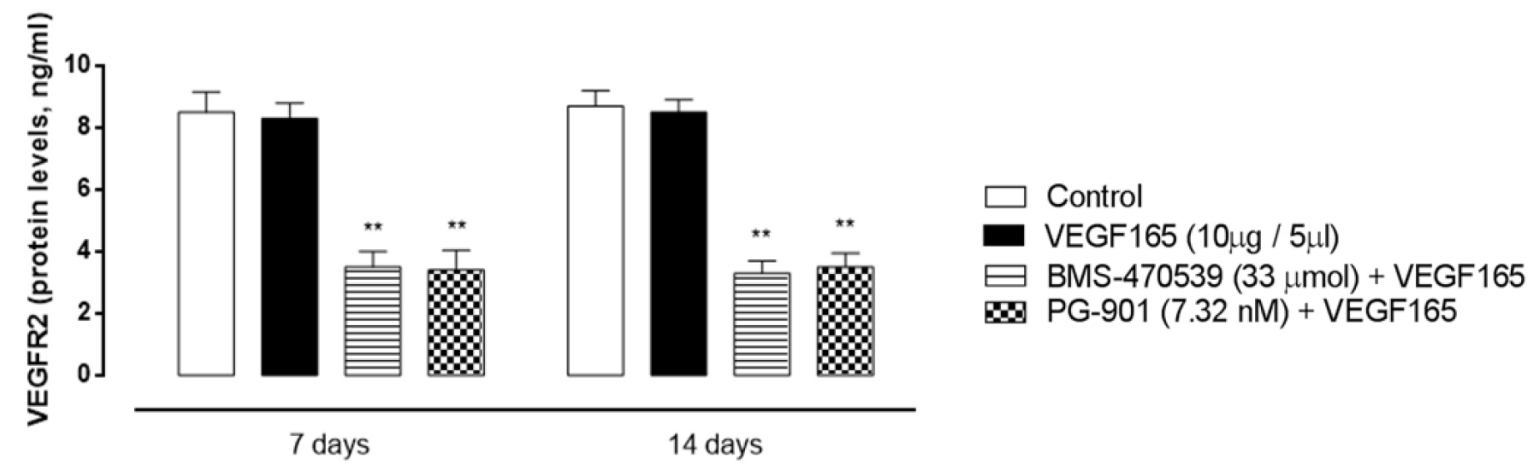

Figure 6. Retinal VEGFR2 protein expression levels (ng/ml) analyzed by Elisa assay 7 and 14 days after VEGF165 intravitreal injection. BMS-470539 (33 $\mu \mathrm{mol})$ and PG-901 (7.32 nM) significantly decrease VEGFR2 protein expression levels at 7 and 14 days, compared to mice receiving saline only. Results are expressed as mean \pm SEM of $=4$ observations for each group. ${ }^{*} \mathrm{P}<0.01$ vs Control.

This paper describes for the first time the protective role of the MC1-5 receptor agonists in a mouse model of retinopathy. The paper shows that a single intravitreal injection of the $\mathrm{MC1}$ receptor agonist BMS-470539 or MC5 receptor agonist PG-901 7 days before and 1 day after the injection of VEGF165 into the vitreous prevents the development of retinal neovascularization as viewed by FAG.

Based on the results obtained in the present study, melanocortins and their receptors seem to be good candidates for treating VEGF dependent neovascularization of the retina having shown pivotal control of the development and progression of new vessels into the retina in the mouse model of retinopathy adopted here, although the single concentration of agonist used is a limitation of any crossmolecule comparison.

Melanocortins are endogenous peptides that possess a wide range of biological activities, including promotion of the resolution phase of inflammation effects in experimental models of inflammationbased diseases [19-27]. Their receptors are ubiquitously expressed into the eye and called MC1, MC3, MC4 and MC5. Rossi et al., in an experimental paper published in 2016 [13] showed that among the four melanocortin receptor subtypes expressed in the retina the $\mathrm{MC} 1$ and MC5 are the two involved in a positive control of diabetic retinopathy. Indeed, the activation of these receptors by specific agonists induces a series of downstream events such as reduction of inflammatory cytokines IL- $1 \alpha$, IL-1 $\beta$, IL- 6 , chemokines MIP- $1 \alpha$, MIP$2 \alpha$, MIP-3 $\alpha$ and M1 macrophage that lead to reduced retinal damage. To this data, we add here a reduced retinal derangement from $\mathrm{MC1,5}$ agonism exerted through reduced proliferation of new vessels in this tissue.

From the molecular point of view we noted that these structural changes were paralleled by biochemical alterations such as reduction in the expression of the VEGFR2 receptor within the retina both at 7 and 14 day time points. The down-regulation of VEGFR2 was caused by selective stimulation of MC1- 5 receptors. Indeed, at the same time points it was not present in the control group treated with VEGF165 only. Thus, neovascularization induced by VEGF165 within the retina is hampered by selective activation of endogenous melanocortin $\mathrm{MC1}, 5$ receptors and reduced VEGFR2 receptor expression.
How this happens would require further investigation, but a novelty is provided here by the strict correlation between functional improvement of retinal vessel form, VEGFR2 expression and retinal expression of the miRNA mir-150. This mir-150, reported to attenuate retinal vascular overgrowth in diabetic mice through a down-regulation of VEGFR2 gene expression [27], is for the first time related to melanocortin $\mathrm{MC} 1,5$ receptors stimulation by the present study. MC1,5 receptors stimulation increases the expression of the retinal mir-150, consequently down-regulating VEGFR2 gene expression and protein levels. This finally improves the early vascular disorders induced by VEGF165 alone. Mirror of this mechanism, CD34 labeling within the retina was reduced. Particularly, CD34 labeling was increased by VEGF165 both at 7 and 14 day time points, confirming the early vascular disorders evidenced by FAG, while it was significantly reduced after MC1,5 agonists.

Noteworthy, CD34 is a transmembrane phosphoglycoprotein well known marker of hematopoietic endothelial progenitor cells (EPCs) proliferation [28], and shown to promote the formation of pathological, invasive vessels during neovascularization in a mouse model of oxygen-induced retinopathy [29]. Interestingly, the differentiation of these cells from the hematopoietic stem cells (HSCs) is associated with the up-regulation of vascular endothelial growth factor receptor 2 (VEGFR2), after which the HSCs are considered endothelial progenitor cells (EPCs), effectively [30-32].

\section{Conclusions}

In conclusion, there is positive interplay exerted through increase of mir-150 between retinal melanocortin MC1,5 receptors and VEGFR2 receptor in the model of VEGF165 induced retinopathy in order to prevent the local neoangiogenesis and retinopathy. From the translational point of view, it is tempting to speculate that the enhancement and reinforcement of the action of the endogenous melanocortins pathway can be a tool to prevent the development of retinal neovascularization, probably by amplifying its antiinflammatory, anti-oxidant and anti-proliferative role. The potential systemic toxicity of the agents used in this study has not been investigated and would require deepening in order to reinforce their efficacy. 
Acknowledgments: The authors are grateful to Professor Paolo Grieco (Pharmacy Department, University of Naples Federico II) for his support and acknowledge the precious funding of MIUR PRIN 2015 project.

Conflicts of Interest: The authors declare no conflict of interest.

\section{References}

1. Miller JW (1997) Vascular endothelial growth factor and ocular neovascularization. Am J Pathol 151: 13-23. [Crossref]

2. Wild S, Roglic G, Green A, Sicree R, King H (2004) Global prevalence of diabetes: estimates for the year 2000 and projections for 2030. Diabetes Care 27: 1047-1053. [Crossref]

3. Friedman DS, O’Colmain BJ, Muñoz B, Tomany SC, McCarty C, et al. (2004) Prevalence of age-related macular degeneration in the United States. Arch Ophthalmol 122: 564-572. [Crossref]

4. Visser L, Singh R, Young M, Lewis H, McKerrow N (2012) Guideline for the prevention, screening and treatment of retinopathy of prematurity (ROP). S Afr Med $J$ 103: 116-125. [Crossref]

5. Veikkola T, Alitalo K (1999) VEGFs, receptors and angiogenesis. Semin Cancer Biol 9: 211-220. [Crossref]

6. Dai J, Rabie AB (2007) VEGF: an essential mediator of both angiogenesis and endochondral ossification. J Dent Res 86: 937-950. [Crossref]

7. Park JE, Keller GA, Ferrara N (1993) The vascular endothelial growth factor (VEGF) isoforms: differential deposition into the subepithelial extracellular matrix and bioactivity of extracellular matrix-bound VEGF. Mol Biol Cell 4: 1317-1326.

8. Ishida S, Usui T, Yamashiro K, Kaji Y, Amano S, et al. (2003) VEGF164-mediated inflammation is required for pathological, but not physiological, ischemia- induced retinal neovascularization. J Exp Med 198: 483-489. [Crossref]

9. Ishida S, Usui T, Yamashiro K, Kaji Y, Ahmed E, et al. (2003) VEGF164 is proinflammatory in the diabetic retina. Invest Ophthalmol Vis Sci 44: 2155-2162. [Crossref]

10. Arana LA, Pinto AT, Chader GJ, Barbosa JD, Morales S, et al. (2012) Fluorescein angiography, optical coherence tomography, and histopathologic findings in a VEGF(165) animal model of retinal angiogenesis. Graefes Arch Clin Exp Ophthalmol 250(10): 1421-8. [Crossref]

11. Badaro E, Novais EA, Abdala K, Chun M, Urias, M, et al. (2014) Development of an experimental model of proliferative retinopathy by intravitreal injection of VEGF165. J Ocul Pharmacol Ther 30(9): 752-6. [Crossref]

12. Andreoli CM, Miller JW (2007) Anti-vascular endothelial growth factor therapy for ocular neovascular disease. Curr Opin Ophthal mol 18: 502-508. [Crossref]

13. Rossi S, Maisto R, Gesualdo C, Trotta M.C, Ferraraccio F, et al. (2016) Activation of Melanocortin Receptors MC 1 and MC 5 Attenuates Retinal Damage in Experimental Diabetic Retinopathy. Mediators Inflamm 7368389

14. Maisto R, Gesualdo C, Trotta MC, Grieco P, Testa F, et al. (2017) Melanocortin receptor agonists MCR 1-5 protect photoreceptors from high-glucose damage and restore antioxidant enzymes in primary retinal cell culture. J Cell Mol Med 21: 968-974. [Crossref]

15. Grieco P, Cai M, Liu L, Mayorov A, Chandler K, et al. (2008) Design and microwave-assisted synthesis of novel macrocyclic peptides active at melanocortin receptors: discovery of potent and selective hMC5R receptor antagonists. $J$ Med Chem 51, 9, 2701-2707. [Crossref]

16. Leoni G, Voisin MB, Carlson K, Getting S, Nourshargh S, Perretti M (2010) The melanocortin MC1receptor agonist BMS-470539 inhibits leucocyte trafficking in the inflamed vasculature. Br. J. Pharmacol 160(1): 171-180. [Crossref]

17. Møller CL, Raun K, Jacobsen ML, Pedersen TÅ, Holst B, et al. (2011) Characterization of murine melanocortin receptors mediating adipocyte lipolysis and examination of signalling pathways involved. Mol Cell Endocrinol 341(1-2): 9-17. [Crossref]

18. Di Filippo C, Zippo M.V, Maisto R, Trotta M.C, Siniscalco D, et al. (2014) Inhibition of ocular aldose reductase by a new benzofuroxane derivative ameliorates rat endotoxic uveitis. Mediators Inflamm 9.

19. Manna SK, Aggarwal BB (1998) Alpha-melanocyte-stimulating hormone inhibits the nuclear transcription factor NF- B activation induced by various inflammatory agents. J Immunol 161, 6, 2873-2880. [Crossref]

20. Martin LW, Catania A, Hiltz ME, Lipton JM (1991) Neuropeptide alpha-MSH antagonizes IL-6- and TNF-induced fever. Peptides 12: 297-299. [Crossref]

21. Bhardwaj RS, Schwarz A, Becher E, Mahnke K, Aragane Y, et al. (1996) Proopiomelanocortin-derived peptides induce IL-10 production in human monocytes. J Immunol 156, 7, 2517-2521. [Crossref]
22. Redondo P, García-Foncillas J, Okroujnov I, Bandrés E (1998) Alpha-MSH regulates interleukin-10 expression by human keratinocytes. Arch Dermatol Res 290: 425-428. [Crossref]

23. Grabbe S, Bhardwaj RS, Mahnke K, Simon MM, Schwarz T, Luger TA (1996) -melanocyte-stimulating hormone induces hapten-specific tolerance in mice. $J$ Immunol 156, 2, 473-478.

24. Slominski A, Wortsman J, Luger T, Paus R, Solomon S (2000) Corticotropin releasing hormone and proopiomelanocortin involvement in the cutaneous response to stress. Physiol. Rev 80, 3, 979-1020. [Crossref]

25. Scholzen TE, Sunderk"otter C, Kalden DH, Brzoska T, Fastrich M, et al. (2003) alpha-melanocyte stimulating hormone prevents lipopolysaccharide induced vasculitis by down-regulating endothelial cell adhesion molecule expression. Endocrinology 144, 1, 360-370. [Crossref]

26. Raap U, Brzoska T, Sohl S, Päth G, Emmel J, et al. (2003) -melanocyte-stimulating hormone inhibits allergic airway inflammation. J Immunol 171, 1, 353-359. [Crossref]

27. Shi L, Kim AJ, Cheng-An Chang R, Ying W, Ko ML, et al. (2016) Deletion of mir150 exacerbates retinal vascular overgrowth in high-fat-diet induced diabetic mice. PLoS One 11(6): e 0157543. [Crossref]

28. Ivanovic Z (2010) Hematopoietic stem cells in research and clinical applications: The "CD34 issue". World J Stem Cells 2: 18-23. [Crossref]

29. Siemerink MJ, Hughes MR, Dallinga MG, Gora T, Cait J, et al. (2016) CD34 promotes pathological epi-retinal neovascularization in mouse model of oxygeninduced retinopathy. PLoS One 11(6): e0157902. [Crossref]

30. Körbling M, Katz RL, Khanna A, Ruifrok AC, Rondon G, et al. (2002) Hepatocytes and epithelial cells of donor origin in recipients of peripheral-blood stem cells. $N$ Engl J Med 346(10): 738-46. [Crossref]

31. Yeh ET, Zhang S, Wu HD, Körbling M, Willerson JT, Estrov Z (2003) Transdifferentiation of human peripheral blood CD34+-enriched cell population into cardiomyocytes, endothelial cells, and smooth muscle cells in vivo. Circulation 108(17): 2070-3.

32. Murohara T, Ikeda H, Duan J, Shintani S, Sasaki Ki, et al. (2000) Transplanted cord blood-derived endothelial precursor cells augment postnatal neovascularization. $J$ Clin Invest 105(11): 1527-1536. [Crossref]

\section{Citation:}

Rosa Maisto, Maria Consiglia Trotta, Settimio Rossi, Carlo Gesualdo, Dario Siniscalco, Franca Ferraraccio, Jorge Miquel Barcia, Francesca Simonelli, Clara Di Filippo, and Michele D'Amico (2018) Intravitreal injections of Melanocortin receptor 1,5 agonists prevents neovascularitazion in a VEGF165 mouse model of retinopathy. J Clin Res Med Volume 1(2): 1-7 